\section{The Advantages of Using a Videomicroscope for Classroom Teaching}

\author{
David J. Hedgepeth \\ Deptartment of Middle Grades Education, \\ Valdosta State University
}

To see what is general in whet is particular, and what is permanent in what is transitory is the aim of scientific though Alfred North Whitehead

Science educators in K-12 schools are often confronted with skepticism when the budgetary needs for equipment and materials come up for discussion. They are expected to make science "interesting", "exciting", "motivating", and "up-to-date", but without investing money in modern technology. It is important to not let this common fascination with novelty, excitement and parsimony, prevent us from realizing the full potential for videomicroscopy in classroom science teaching. In addition to their "gee, whizl" aspects, videomicroscopes offer a number of instructional advantages that make them worth the money, and I will present some ideas about how to use and configure a videomicroscope for classroom use.

One great advantage of videomicroscopy is that the image produced by a high quality videomicrosoope can be remarkably bright and precise. Focusing the image can be simplified, since eyeglasses can be worn by the different viewers, and the stage manipulation can be more intuitive, since the operator is able to watch what is happening in a more normal setting on a screen. These characteristics are especially important when considering the needs of students with disabilities. Students who have low or reduced vision may have great difficulty in effectively viewing a specimen though a typical school microscope. The full view on a monitor can be much more easily seen, especially by those students who only have peripheral viewing or low vision.

Students who have physical disabilities or coordination problems may not be able to effectively manipulate the controls of a typical compound microscope. This may be due to an inability to focus visually, or inability to control the movements of the head, eye, and hand, simultaneously, while still concentrating on the specimen in the field of view. Students with mental disabilities may have difficulties with the instructions needed to set up and follow a microscopy lesson without assistance; however, this does not mean that they would not benefit from access to some of the same educational experiences provided to other students. There is a great range of special needs, from the learning disabled to the emotionally handicapped; the teachers who work with these students know their scholastic level and can choose the kinds of activities that would be effective with them. It is possible that watching a swimming Volvox can cover many diverse objectives.

One of the other ways in which videomicroscopy can meet the needs of diverse learners is by allowing group observation and discussion. Rather than one person relaying their observations back to the others, taking turns at the eyepiece, refocusing, and then the inevitable search for the same remarkable specimen or mysterious artifact, the whole group can work collaboratively to identify, investigate and report their findings.

The interpretation of the dynamic scenes one finds under magnification is also a great opportunity to use the unique features of the videomicroscope. For example, when fresh pond water samples are being examined for protozoans and metazoans, questions like "what is that thing doing?" or "what do they eat?" can start the inquiry process as students reason together about what is happening, and what it means. This social process of negotiating the meanings of things is considered by many science educators to be an essential step in the development of students' understanding of the world around them.

Another way in which a videomicroscope can enhance and expand learning is by providing a visual context for posing questions, showing relationships, predicting, making hypotheses and other tasks usually done by talking or writing about microscopic specimens. These can now be done more visually by pointing, marking, gesturing, using the actual image as a text rather than a verbal rendition of it.
One of the frustrations of working with live micro specimens is their evanescent nature. By recording observations on videotape (or digitally), students can create a record of their observations and include the visual data either to support their conclusions, or to extend their questions. By making observations of the same specimens over time, or by comparing recorded observations of many specimens, students can look for patterns.

Also, unique events caught on tape can be shared with others. This serendipity can reinforce the understanding that discoveries depend on preparation and careful study, rather than simple chance. To paraphrase the phenomenological question, "If an interesting and unexpected microscopic event happens, and no one records it - did it really happen?"

One other advantage of recording videomicroscope specimens is that school schedules and cultures both are subject to change. If you had great specimens on Friday, will you still be able to find them on Monday? Or, if some lab groups are not able to complete their activities, you can show them the results from previous work. Also, some VCR's will allow time lapse photography. This is a great approach for low magnification of specimens where focus is not so critical, since over time the specimen may shift, or develop out of tight locus. These sequences can be a very powerful way to show the dynamic character of life on a micro level, even when we aren't watching.

Students today live in a culture of technological products of all types. Every aspect of their lives involves electronics, video, and computers. Receiving information in this context is very natural to these students, and this approach is consistent with the real work which is being done in research labs, industry, and universities. Using these modern tools in learning makes a connection between the classroom and the scientific and technological society that students live in now, and for the future.

What is the best videomicroscope system for a classroom? Some people would argue that the best system is one that you actually have! Even though yours may not be an "ideal" or "state-of-the-art system", it may actually be the best one for your needs. Many of the features of research microscopes are only useful in conjunction with other expensive equipment, or difficult techniques. The multitude of knobs, dials, and adjustments means that lots of things can get out of whack (and will, especially, with student use); refocusing a befuddled precision microscope may not be the thing you most want to do six times in the middle of class. Also, if the equipment is administratively dubbed too expensive to use for fear of messing it up, then it probably will sit in the closet until everyone forgets how much it cost.

Fortunately, video cameras are getting less expensive, more compact, and are available with more features. Color cameras are now in the same general price range as black and white, and offer many advantages. A good camera is important, but unless you have a high resolution monitor to display the image, then a more expensive and better camera may not result in any better picture. Other pieces of necessary equipment include the camera adapter, and the "mount" or microscope head. The camera adapter fits the camera to the microscope head. The microscope head can be the simple monocular head that is standard on many scopes. One problem with this arrangement is that it requires you to focus using the monitor, and you cannot easily switch to and from standard viewing.

Even with a very good system, more detailed resolution and color can be seen without sending the image through all the additional gadgets to the monitor. Some heads include a switching device so that you can selectively either focus using the eyepiece, or view the specimen on the monitor and some will let you do both at the same time. If you have binocular eyepieces, it is possible to mount the camera on one, and focus with the other. However, if your microscope has the eyepiece tube (or tubes) inclined at an angle, it may be difficult to balance the assembled equipment with enough stability to allow viewing without excessive vibration. A "trinocular" head is highly desirable for a videomicroscope system. This type of head has a straight upright tube for the camera mount, and binocular eyepieces for individual viewing and focusing.

A color monitor is almost mandatory for classroom use with students, and should match the capability of the color camera. Whether you are viewing living specimens or stained slides, many details, and much of the essential beauty and interest will be lost in monochrome especially for beginning students. Once again. the monitor should match the capability of the rest of your system. If it cannot show the same level of detail as the camera and microscope send to it then it becomes the limiting factor. This applies to the video cassette recorder as 
well. It is certainly possible to operate the videomicroscope and monitor without having a VCR. But if it is possible to include one in the setup, then you will have great opportunities to expand the role of microscopy in your lessons.

Finally, with the addition of a computer that can digitally process your videomicroscope images, you can introduce powerful instructional features of image processing software. These possibilities range from the capability to add labels and grids to images on the screen, to real time measuring of size and area, color enhancing, coding, networking, and integrating processed images into multimedia formats. There are a number of software packages specifically designed for processing videomicrosoope images, and we can expect to see more of them as faster and more powerful microcomputers become widely available. Finding the right one for your needs will probably

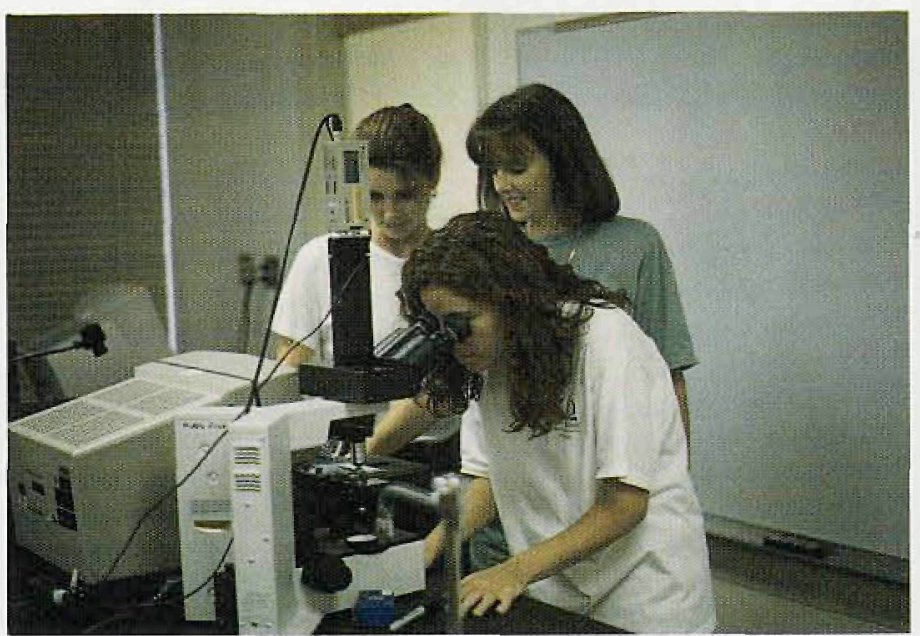

Education Students Discussing a Videomicroscope Image

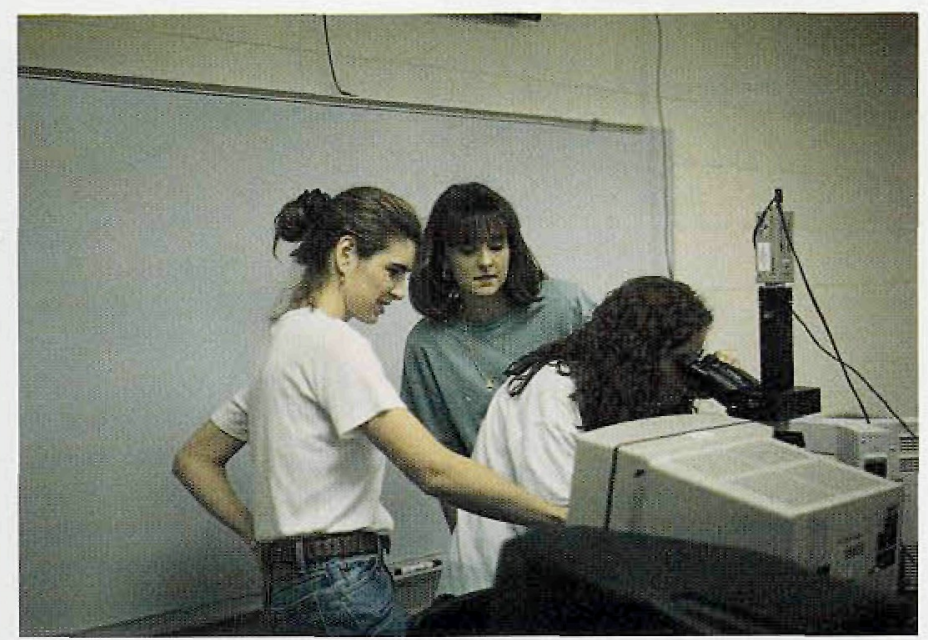
compatibility.

College students who are preparing to be public school science teachers need to develop skill in using a broad range of tools in the classroom and laboratory. Multimedia presentations, distance learning, and the information super highway, are radically changing people's ideas about what teaching should be like in the 21st Century. However, most of the instruction that future science teachers receive about microscopy does not make use of recent advances. Rather than reserving these wonderful new devices for research only, even greater benefits can come from providing teachers with both the technical know-how and effective pedagogical strategies as they share amazing microscopes with the next generation of scientists and science literate citizens. require shopping around and testing demo software packages for capability and

\section{Microscope}

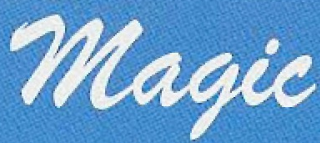

\section{Introducting SEMICAPS "GENIE" Digital Imaging System for Windows ${ }^{\text {T.. }}$.}

Capture high resolution digital images from any analog or digital SEM, TEM, or Optical Microscope.

GENIE will improve your productivity...

* Smart archival system with keyword searches and retrieval * Automated report generation with user defined Print Templates * Automated data entry with Keyword Macro

\section{alsa Introducing...}

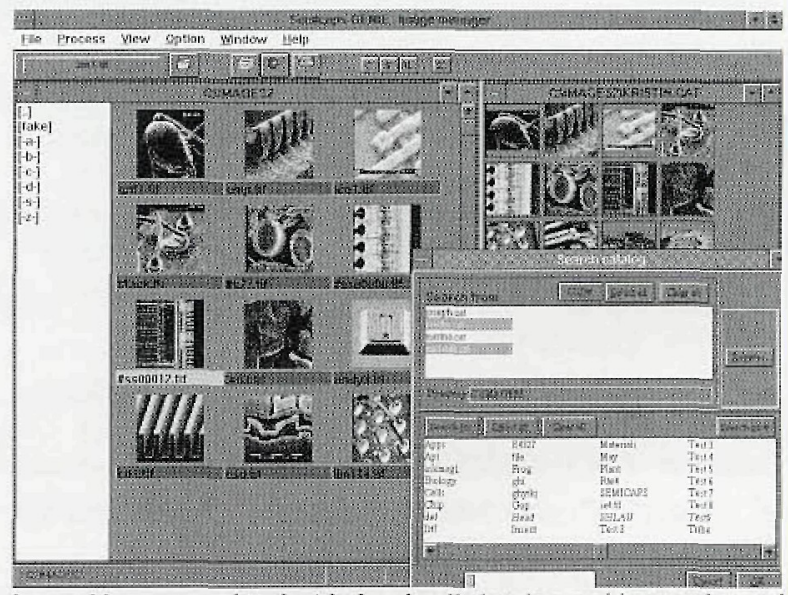

Image Manager archival with thumbnail sketches and kevword search

the SEMICAPS CL300, Monochromatic Cathodoluminescence(CL) Imaging and Analysis System. This detector provides unparalleled collection efficiency and backscatter electron rejection. Retrofit the CL 300 to any conventional or FEG SEM and receive the highest possible spatial resolution for materials charaterization using CL. 
$\checkmark$ American Type Culture Collection (ATCC) Workshops:

July 7/10 '97: Theory and Techniques for Extremophile Research

Nov 4/7 97: Microbial DNA Fingerprinting

Nov 10/14 '97: Advanced Recombinant DNA: Techniques and Applica tions

Nov 17/20 97: Polymerase Chain Reaction (PCR) Applications/Cycle

DNA Sequencing

Sept 8/10 97: Cell Culture and Hybridomas: Quality Control and Cryopreservation Techniques

Sept 15/16 '97: The 15th Annual ATCC Biotech Patent Forum

Sept 17/19 '97: Cytogenetic and Fluorescence in Situ Hybridization

Sept 23/26 '97: Fermentation Microbiology

Oct 6/9 '97: Hybridoma Technology and Monoclonal Antibody Product Development

Oct 15/17 97: Microscopy/Photomicrography

Oct 15/17 97: Diagnostic Virology

Oct 22/24 97: Downstream Processing, Recovery and Purification of Proteins

Oct 27/30 97: Freezing and freeze-Drying of Microorganisms 4364

INFORMATION: (800)359-7370, Local: (301)231-5566, Fax: (301)816-

$\checkmark$ July 6/9 '97: CRYO '97 - Low Temperature Microscopy and Analysis \& The Application of the Microscope in Life Sciences Cells, and Signaling Including FLOW. (Royal Microscopical Society) Univ. of York, RMS: $+44(0) 1865$ 248768, Fax: $+44(0) 1865791237$

$\checkmark$ July 20/25 '97: STM '97 Hamburg, Germany, +49-40-4123-5244 Fax: $+49-40-4123-6368$

$\checkmark$ July 21/24 '97: INTER/MICRO-97 (McCrone Research Institute), Chicago, IL. Nancy Daerr: (312)842-7100, Fax: (312)842-1078, eMail: ndaerr@mcri.org

$\checkmark$ Aug 4/8 97: Denver X-Ray Conference. Steam Springs, CO. Carole Collier, email: denxrcon@de.edu

$\checkmark$ Aug 9/10 '97: Multi-Photon Excitation Symposium and Workshop (Univ of W//Carnnegie Mellon Univ/MSA). Cleveland, $\mathrm{OH}$. Dawn Volkman: (608)265-3083, dvolkman@students.wisc edu

$\checkmark$ Aug 10/15 '97: Microscopy Society of America 55th Annual Meeting - Jointly with the Microbeam Analysis Society. Cleveland OH. MSA Business Office: (800)538-3672. Fax: (508)548-9053

$\checkmark$ Aug 25/29 '97: Computers in High-Resolution TEM (NCEM Summer School), Berkeley, CA. Gretchen Hermes: http.ncem.lbl.gov, (510)486-5006, Fax: (510)486-5888, eMail: GHermes@|bl.gov

$\checkmark$ Sept 7/11 '97: 111th AOAC International Annual Meeting \& Eposition. San Diego, CA. (800)379-2622, Fax: (301)924-7089

$\checkmark$ Sept $7 / 12$ '97: 11th CONF ON SIMS. Santa FFe NM: (505)989 4735, Fax: (505)989-1073

$\checkmark$ Sept 22/25 97: International Conference on Texture and Anisotropy of Polycrystals (TU Clausthal, Germany). Robert Schwarzer: Fax: (+49)5323-722340, eMail: schwarzer@tu-clausthal.de

$\checkmark$ Sept 25 '97: Computers in Microscopy (RMS) Cambridge, UK Rebecca Morden: eMail: info@rms.org.uk

$\checkmark$ Oct 5/8 '97: Multinational Congress on Electron Microscopy. Portoroz, Slovenia. http://WwW2.ijs.si/-k5www/MCEM97/index.html

$\checkmark$ Oct 8/16 '97: Optical Microscopy and Imaging in the Biomedical Sciences (Marine Biological Lab) Woods Hole, MA. Carol Hamel: (508)289-7401.

$\checkmark$ Nov. 16/20 '97: Eastern Analytical Symposium. Somerset, $\mathrm{NJ}$ (302)738-6218, Fax: (302)738-5275.

$\checkmark$ Dec 1/5 '97: MRS Fall Meeting Boston, MA. MRS: (412)367. 3004, Fax: (412)367-4373
Opportunity to work actoss research, development, and manufacturing technology sectors of Corning Incorporated, a global company. Primary responsibility includes characterizing materials for their optical and microstructural properties. The scientist wil utilize a variety of instrumentation including: polatized light microscopy, interferometric microscopy, image processing and analysis and optical imaging and spectroscopy in the near-field, as well as, developing newer measurement methods. The position involves both working individually and on cross-functional teams.

Minimum Requirements:

- Ph.D. in Optical Physics, Materials Science or Geology

Optical microscopy

Optical image processing and analysis

Good communication skills, creativity, flexibility and self-drive Desired Experience:

Optical Spectroscopy as applied to Near-field Optical Microscopy and Spectroscopy (NSOM)

- Optical crystallography or Polarized Light Measurements Glass and polymer science

Corning Incorporated is a producer of technical glass, ceramic, and polymer products, and has a long-standing reputation of advanced scientific invention and development of telated materials. Inquiries and resumes should be submitted to:

$$
\begin{aligned}
& \text { Karen J. Mortissey } \\
& \text { Corning, Inc. }
\end{aligned}
$$

Chatacterization Science and Service Directorate SP-FR-01-8

Corning, NY 14831

Tel.: (607)974-3385, Fax: (607)974-3385 eMail: Morrissey_K@Corning.com

Corning is an Equal Opportunity Employer EEO/MF

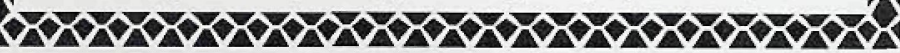
TECHNICAL SALES REPRESENTATIVE
SCANNING PROBE MICROSCOPES

Park Scientific Instruments (PSI), a world leader in the rapidly growing field of Scanning Probe Microscopes (SPM) is looking for a technical sales representative for the upper midwest territory ( $\mathbb{L}, \mathbb{N}, \mathrm{MI}, \mathrm{WI}, \mathrm{MN}$, etc.). Applicants must have a B.S. or higher degree in a physical science and a minimum of 3-5 years sales experience in analytical or other high technology products. Familiarity with microscopy and image analysis, life sciences, or materials science is a plus. We want a self-motivated, success oriented experienced sales representative with a high degree of independence and a strong desire to win. The successful applicant will be provided an attractive base salary and the opportunity for high additional income through a sales commission program, an automobile allowance and other liberal company benefits.

If you meet these qualifications and are ready for a rewarding and challenging career, please mail or FAX your resume to

Lance Hellinger

Park Scientific Instruments

1171 Borregas Avenue

Sunnyvale, CA 94089-1304

Tel.: (408)747-1600, Fax: (408)747-1601 

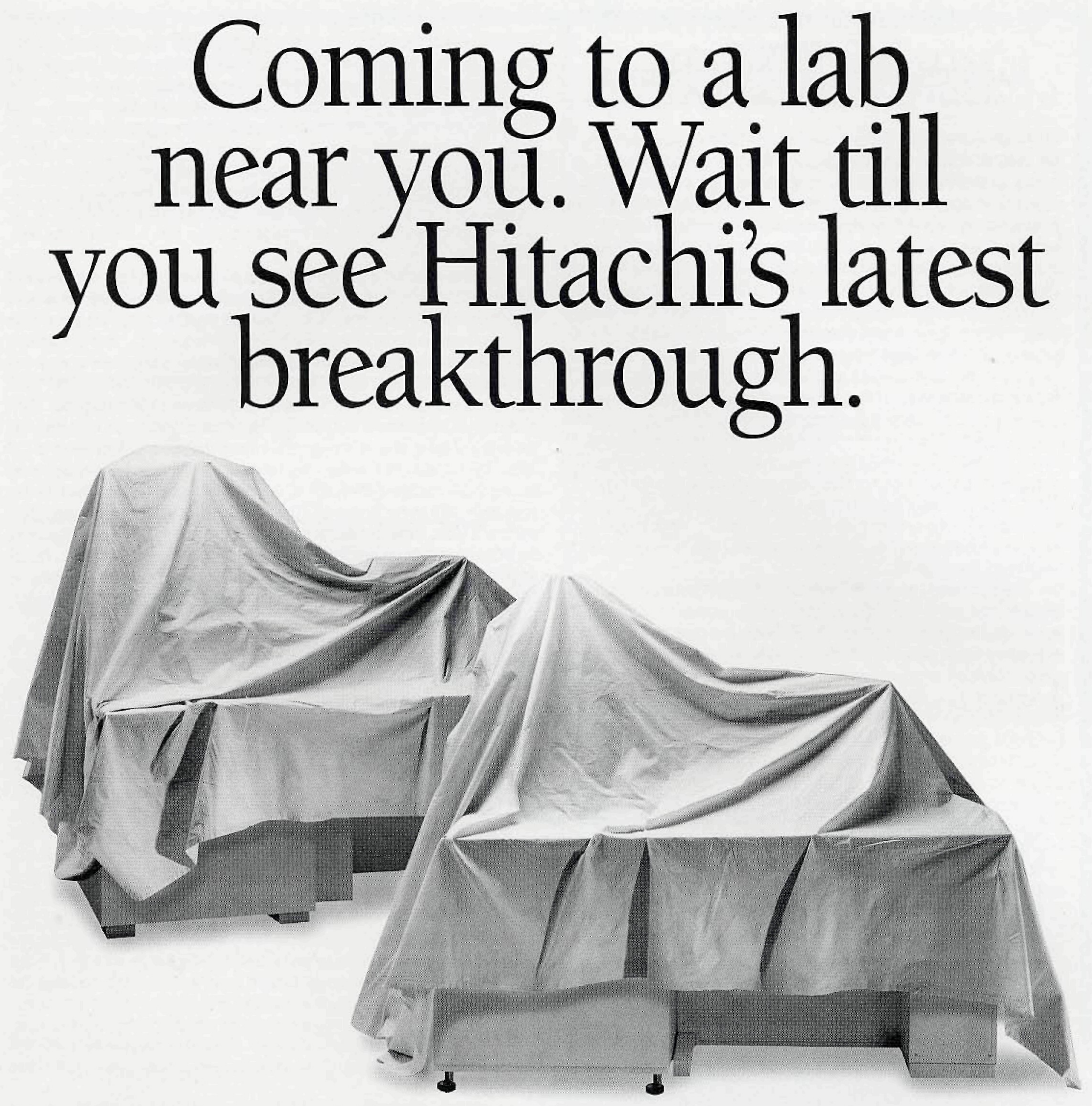

\section{HITACHI}

SCIENTIFIC INSTRUMENTS

Nissei Sangyo America, Ltd.

755 Ravendale Drive

Mountain View, CA 94043

(800) 227-8877

E-mail: sidsales@nissei.com

www.nissei.com

25 West Watkins Mill Road

Gaithersburg, MD 20878

(800) 638-4087

Circle Reader Inquiry \#10 